\title{
THE FUNCTION OF STATEMENT IN CROWD MANAGEMENT
}

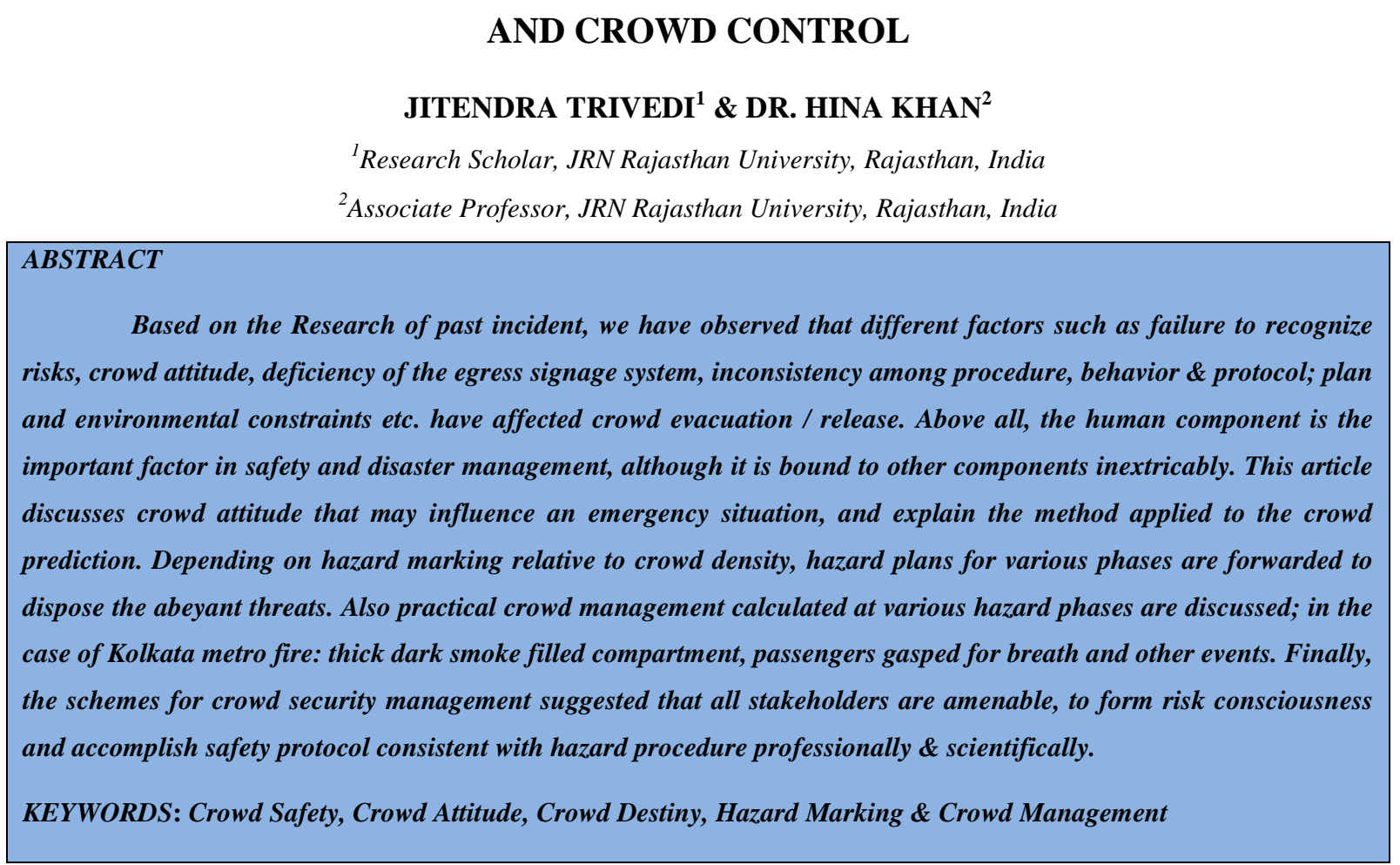

Received: May 05, 2019; Accepted: May 26, 2019; Published: Jun 14, 2019; Paper Id.: IJBMRAUG20191

\section{INTRODUCTION}

In current years, disasters such as accidents in public areas have occurred from time to time, which have resulted in a shock and taught us painful lessons. Numerous samples aggregated from many occasions or events when different celebrations or sport functions in different city or state take place, which create the security risk. During function it has been witnessed when the crowd density profoundly increase to a specific level, the overcrowding brings common influence and huge demand between public. In such case if someone drops or falls, he/she may have no time to take action and even no place to stand properly. At that particular phase people may be pressurized to step on others while blandly managing to avoid the "Domino effect"; that is falling down by the push from each other. The order of the crowds may get down when the number of respondent put themselves at a specific point, which is not about the peoples morality or quality, but is aroused by the normal interaction of people. Or if the crowd pass converges at the bottleneck areas, or reverse cluster and differently gathered joined, stampede incidents are more precisely to happen. It is not easy to specifically note the responsibility that everyone has, and normal communicable modes cannot function. Procedures are abstract while loss of emotional control in crowds is human nature. The "civilized" people in the crowded position are irritable, even offensive, and vulnerable to misconduct, although other component such as the defect of function area management has been also proved to onset the public security crisis. Complex components brings about the huge troubles of crowd security management in emergency programs, moreover, the human component, unsurprisingly, enlarge the program 
hazard eventually. Therefore, acknowledging human actions in crowds, discussing the content, alternating and deciding on the use of possible intervention mechanisms have become undisputedly indispensable for crowd security management.

\section{REVIEW OF LITERATURE}

Over the past era, although crowd research and crowd management functions have advanced and enhanced greatly to give support to crowd managers in preparation and during a crowd program, much more is required. For instance, crowd actions structure landed in science imitation from various fields involving psychology, sociology, theoretical physics, applied mathematics, artificial intelligence and computer science have been founded to support stakeholders better understand crowd actions, but only few have been actually utilized to guide in crowd management, maybe partly because crowd actions are unpredictable; while some structures either forming in various or varying forms are extremely formal or implicit wisdom, some do not conform for utilization. Paralleling the technological equipment and project about crowd organization and venue outlay have been provided for specific aspects of crowd management, but most of them are not being regularly used. A conventional gap among crowd research and crowd management function is indispensible, and more functional decisive support access is required to produce for crowd security management.

India is very familiar to large gatherings or crowded places. Many festivals and occasions like DurgaPooja, ChathPooja, KumbhMela, Ramjan, RathYatra and Hats and rallies attract many people and devotees. Such events attracts huge crowd which creates chaos and thus leads to a mishap. As per national crime record bureau a sum total no. of 4216 incidents of stampede have taken place in India between 2008 and 2018.

\section{RESEARCH OBJECTIVE}

More of the crowd related disasters are occurring during religious festivals. Such incidence commonly takes place in usual events like musical concerts, exhibitions, firework display, sporting events and places like malls, Cinema Theater, market places etc., so it becomes necessary to set up procedure to manage the crowd considering the safety and security of lives, property and rights of all people and to maintain law.

\section{RESEARCH METHODOLOGY}

In this paper, it is discussed as how crowd management is being functioned and an approach in which it can be managed in a better way. The framework is organized by first providing insights into the individuals' and the groups' behavior features in following section concentrating on crowd density, the appropriate operation support such as its supervision, estimation \& grade are explained. Depending on the analysis crowd management strategies are also proposed. Practical measures for crowd management at various hazard phases are examples in a case of various disasters.

\section{Investigation of Crowd Actions Features}

Individual's actions in the crowd show the features of strong possibility, continuity, and even loss of the volition to manage them in the program. For instance, common people move ahead smoothly in the beginning. If crowd makes personal movement slow and complex, they would stop and go; but then chaos might appear as crowds become huger. Crowd actions have been presented in below three quotes. 


\section{Crowd hurting and Dense Actions}

Unexpected accidents may sink crowd into the scare, or even put crowd forward to hurting each-other which further results in emergency condition. At such time people's formal and conscious personality gets deeply altered and is replaced by the unconscious and irrational actions; unless there are specific strong social effects occurring around them such as leaders who can make decisions for the group. Otherwise, person tends to give up their own beliefs and follow others under the pressure in crowd. In reality, it is not easy for people to respond to all the data available at the site to create correct judgments in the emergency so that the conformity is easier to arise during the evacuation. Blind conformity may break the balance of the flow and crowd incidents like stampede or accidents occur.

\section{Concluded Theory and Fast is Slower Impact}

Concluded theory hypothesized that persons in trouble are able to create reasonable decisions (although if it is limited) and also conduct accordingly to achieve excellent output and aims in this study. Moreover, the person's optimum actions always doesn't result as the best alternative for the group, and even sometimes output is the worst. For instance, in fire incidence, to cooperate with other surroundings and to queue up are most likely to get advantage for the whole group to escape, thus an individual's opportunity to leave dangerous facilities enlarge. Unexpectedly if someone pushes, other persons may start to feel that if they do not follow the push, their turn to escape will be threatened, so after consideration the persons' behavior modify out to join the move competition, push other, and try to make every effort to get out of the dangerous area rapidly. If everyone moves fast, the effect of robbing and squeezing will decrease their efficiency. The evacuation time displays a possibility to minimize in the starting and then an enlarging trend with the walkers' expecting speed upgrading. Subsequently, the crowd should be advised to calm down and make the escape orderly to discard the blind movement and inflow the fast is slower impact.

\section{Curving Phenomenon and "Domino Impact"}

The inlet, famous destinations and the outlet are marked to be the bottleneck by public's movement. When a crowd comes at these places, curving phenomenon may occur. For instance, when in emergency a number of individual try to release consequently form the outlet, curving, clogging and draw back charge burst can be observed. At this stage the bottleneck places become so important that once a tiny accident such as someone's dropping, allotting or rumors occurs, the "Domino Effect" starts as interaction pressure transmitted between the congestion points. The narrow edge of bottleneck areas limit crowd movement so that a jam arises. And the disaster may occur due to competitive actions. Three components determine the congestion degree and the release speed at the bottleneck; serious consequence outputted from failing to evacuate fast, the available releasing time and the group size. Therefore, taking suitable actions to decrease the number of anxious people to free available space to alleviate the creation of the curving queue and then clear away the standing at the bottleneck.

\section{Overlook for the Crowd Density}

In any program or occasion accumulation of public is a process happening due to the large number of participants. The efficient monitoring is considered as a subsequent event of security guarantee. It is important to obtain the data about the features of participants, functions facility status, the security arrangements and the surrounding environment, basically the information from essential area such as stairways, exits, entrances, narrow passages, transports, residence or the convergence of two opposite crowd flows etc. where trickle accidents \& events might onset terrible incident. So intensive 
observing is required to be achieved to supply actual - time components (the crowd density and speed in observing areas) by following and investigating the rolling objects' actions.

- Crowd density is basically featured by the number of individual fitted in per unit area. The greater crowd density basically means the higher degree of population aggregation and also the larger security hazard of the throng. Population density chiefly relies on intelligent video surveillance system for actual time observing of groups.

- Statistical modeling of the pixels, an intuitive and effective procedure, is the first to be adopted to identify population behavior. The common idea of the pixels statistical features is that the greater crowd density reflects the denser foreground pixels and edges ones extracted from the pictures, and the larger occupation proportion in the images.

- The personal quality troubles in high density crowd can be solved by texture examination which is featured by high accuracy. In texture examination procedure, videotapes are separated and correlated for feature extraction. But it cannot fit the low and high population density estimate at the same time, and the huge amount of calculation is not conducive to an actual time video observation structure.

- The different states of the crowds in the context of different congestion densities were explained in the initial work. Integrated with the relative regulations and practice experience from calculating the movement capability of persons in the area of 1 sq. mtrs, crowd density can be graded into 4 classes.

\section{Strategies of Crowd Management}

The effectiveness of the crowd management relies on good arrangement in various area such as prejudgment on the participants, supervision of abeyant threats, the sufficiency of contingency layouts, execution ability and strength of the organizers, the capability of public conversation and data conversation, reasonable division and arrangement for field staff members, the reasonable sign layout, and services for special groups involving the old, children, ill or disabled public and pregnant women, etc.

\section{Analyzing the Sample in the Event}

To predict the ability of specific site for adjusting people, any location has its specified ability to adjust people. Disasters may occur when the ability is overloaded. The capability that a location can afford refers not only to the sum ability of selected specified location, some bottle neck regions where incidents commonly occurs, or some precise permanent or temporary apparatus, but also the endurance management, and command capability of relative security personnel. To check and analyze the inflow and outflow of crowd density, video surveillance and sensor system plays important role.

Considering the formation of sample, the formation of sample is an important component that influences the stability of public functions. Samples are different from one another in features, education status, and personal incidents \& experiences and so on; what's more in different areas the formation of them is complex and different. Forecasting formation of sample in advance can reinforce the pertinence of management plans and the effectiveness of contingency layouts, and permanently, support in dealing with contradictions caused by traditional difference among the sample from varied background. In contemporary, the function organizers are able to produce excellent services in such aspects as traffic, food, and water etc. and take more appropriate conservation steps, if they are aware about the characteristics of 
sample ahead of time. Such access as building up audience management system according to the information from prior years, managing surveys online or offline, accomplishing register system in the procedure of ticket purchase are beneficial to gain the data of possible sample.

For forecasting the suggestion of sample, the time and space segregation of sample in an event can be approximated by historical statistics and mathematical calculation that is involved in the regularity of crowd change, and the allotment of people. Such components as seasonal modification the components of actions and the habitants' culture may have a huge effect on the time allotment of sample while the formation and sequence of activities influences their space allotment. People's possible collecting place, coverage region and the duration of their collecting can be estimated in advance to plan for some peak moments, aim on the bottleneck areas and arrange excellent security measures.

\section{Crowd Management Plan}

It always true that "Precaution is always better then cures". Therefore to make the people aware about crowd management the national disaster management has issued a guideline on "Managing crowd at Events \& Venus of mass Gathering" which supply adequate data concerning crowd management. Roles and responsibilities of all the stakeholders have also been explained in the guidelines.

License, Registration, Approval and permission are mandatory for stage preparation by the venue owner and suppliers to organize the event. The granting authority/ foundation may verify the data provided by the organizer.

Approach of Hazard, Risk and Vulnerability Assessment at the event area produces a platform for preparing for all the stages of disaster management. Following layouts are a part of this strategy :- location of the event, History of location \& event, Time phase of event, list and numbers of visitors, contact information of the event organizer, status of entry, exit, emergency rescue area, Holding region, evacuation route, parking and shopping area. Moving its step further towards safety and security, it also includes the layout as such of public approaching system, entry and exit, fire precaution, electrical installation, Traffic management, status of food vendors and their equipment, emergency power status, Note on suspicious persons, unattended packages are also to be done.

\section{Initial Warning Status in Crowd Security Management}

Initial warning system, an intelligent system that runs automatically points out to management personnel of locations conditions accordingly by observing information, it is an anti - risk program that makes an alert for relevant steps to be taken in advance. Initial warning status data is used by various phases of risk, and then to advance suitable control plans. Initial warning phase and the relevant advice and steps taken can be explained in below table.

Table 1: Layout for Varied Hazard Phases

\begin{tabular}{|c|l|l|}
\hline $\begin{array}{c}\text { Hazard } \\
\text { Phase }\end{array}$ & $\begin{array}{c}\text { Warning } \\
\text { Indication }\end{array}$ & \multicolumn{1}{c|}{ Layout for Varied Hazard Phases. } \\
\hline I & Red & $\begin{array}{l}\text { Contemporary \& adequate emergency vehicles } \\
\text { and manpower are confirmed to help for the } \\
\text { rescue. }\end{array}$ \\
\hline II & Orange & $\begin{array}{l}\text { Connection among people become essential, all } \\
\text { stakeholders take part in evacuation approach. }\end{array}$ \\
\hline III & Yellow & $\begin{array}{l}\text { Evacuation is suggested. The congestion is being } \\
\text { disposed. }\end{array}$ \\
\hline IV & Green & Security personnel are required to patrol the scene. \\
\hline
\end{tabular}


For hazard Phase 1, the possibility of security hazard concerning as stage I is much huge. The entire individual in the location should be rescued safely and securely in very less time. Compulsory evacuation is carried out. Be silent and make crowd calm and guide it. Continue to rescue the individuals.

- At the hazard phase 2 the approach is overcrowding. Individuals are stepping by the involuntary movement due to rush. The pressure from the interaction among the crowd can be experienced. Moment and condition is dangerous and connection with the crowd gets important to ignore any unusual events. All the stakeholders cooperate to take part in rescue guidance.

- As for hazard phase 3, more individuals throng into the notable regions, congestion appears at times, and specific types of danger noted. However, the situation is still manageable, and the risk is barely acceptable.

- For the hazard phase 4, crowds move smoothly and security personnel are not required to take strict control steps, but only a small number of them are required to petrol the scene, arrange and suggest posts reasonably and pay attention to modify the crowd.

\section{Crowd Diversion Steps}

Depending on the crowd density surveillance, initial warning mechanism, prediction process; the information about the crowds are gathered and required to be announced to the staff included in the event.

Selecting reasonable paths: - when selecting paths to segregated the crowd, the stationary flow and the capacity of facilities to contain people, as well as some precise situation (Weather, types of emergencies, and road situations) should be considered to remove the unreasonable section so as to avoid the obstacles that may affect rescue achievement. Improper infrastructure is one of the drawbacks that decrease the crowd capacity and slow the speed.

Both disorderly framework and unreasonable segregation increase the hazard in an event. Searching the rescue traffic becomes the secondary situation to keep the sample safe. Smooth traffic can be guaranteed by adding striking road maps, planning business streets and stalls, managing peddlers and setting up service areas. Medical facilities and professional staffs should be provided as a routine work.

Discharging data timely - data sample of the rescue guidance at all times and releasing data about the location situation on time has been always important in any event. Note that in few disasters examples, it was seen that people at the back of the sequence couldn't get any idea of what had happened at the front and that resulted in unsuitable events such as push and shove, precipitated them into tension etc. which finally led into grave significance. Also, as declared above, most people tended to sink into the panic and be at loss in emergency, and just follow others without response to all the data in the location. Therefore, notifying people of routes, cues and signs at exits and entrances and other data can reduce their misunderstanding about the conditions, provide them important message and allow them to make appropriate decisions. Moreover, rallying on the forecast information, organizers can give sample some suggestions to influence their alternative involving the time and path to go out, the members, paths and items to visit etc; So, that the collecting pressures at the equivalent places and at the equivalent time can be relieved.

\section{Incident Response Plan}

The theory of "Incident Response system" in overall management of event has been found to be an effective and efficient management system, all the stakeholders should function their actions and responsibility as per the response plan. 
Roles and responsibilities of all the stakeholders like police, health, PWD, forest, fire services, Home Guard and civil defense, Red Cross, Public Health and Engineering may be explained transparently.

Process for the activation of plans: the incident response plan may be activated to control the incidence as and when needed. The event will display the urgency and necessity of the plan.

Emergency operation centre may be established at a central position so that immediate movement can be performed. It should be established under the local administration with representatives from the line departments.

Media should need to act responsibly at the place of mass collecting. Media should spread the data of Do's and Don'ts. During the event it is required to have cooperation of the media and the governmental and non - governmental agencies.

\section{A Special Case}

Take a metro station of Kolkata as an example. With the advancement of urban traffic, subway has become a competitive alternative for people to travel owing to its benefits of convenience and efficiency. Some subway stations maintain heavy traffic throughout the year and the passenger flow consequently upgrades specifically in the holidays, which puts to the crowd aggregation in a huge level and stampede incidents or other disasters are prone to happen.

\section{DATA ANALYSIS}

Efficient hazard evaluation and initial warning method are important to pre-control the possible disaster triggers in busy subway stations. First, it is crucial to adjust and manage the apex crowd flow limit of rescue passages. The capability of each location should be estimated to predict its limitation. The bottleneck regions also require to be considered due to a blockage arises from alternative actions. Meanwhile, whether the exit has place or not also has effect on the stoppage and flow of crowds. The framework of the focal point can be modified to adjust the sample to move freely. Secondly, it is deemed that crowd density, the speed of movement and people's psychology; all have important effects on rescue efficiency. Crowd in any region where each person covers lower than $0.26 \mathrm{~m} 2$ may be not safe; it leads to squeezing, tripping and causalities. So crowd density is observed in actual time and reliable data is released to crowds in time to ignore the loss of rational actions. Third, it's crucial to measure the formation of sample depending to the entrance ticket data system. Environmental component such as temperature, sound and light are formed to comfort people's psychological reaction. Last but not least, a manager who is aware about the location and framework precisely should be effective and appropriate; and experienced guards, safety apparatus and rescue passages must be ensured to rescue the sample.

\section{RESULTS AND CONCLUSIONS}

In any event, all stakeholders are amenable to form hazard consciousness and grasp the basic knowledge and skills to ignore hazards, safeguard themselves and rescue properly and safely in an event. The discussed hazard layout should be studied out in advance to confirm the data about the crowds; ways and processes in various situations should be notified to everyone included in the event. It is extremely indispensable to remove all hidden threats when solving for the event, and connect with and then control the crowd efficaciously during the procedure of the event. Crowd management is important to construct a participatory access with the active advancement of different stakeholders, local authorized individuals, and the community to confirm smooth conduct of events of mass gathering. Crowd management can make difference depending upon a location, type \& situation. Creating strategies for crowd management layout should be an 
exercise which involves the troubles, challenges, venues \& events of mass get- together. The plan should aim on religious places, and transport places with support of the local reliable authority and stakeholders.

\section{ACKNOWLEDGEMENTS}

Authors are greatful to JRN, Rajasthan University, Rajasthan to carry out this research work. Authors are also thankful to Dr. J.G. Pandya and Dr. Manoj Pandya for their support to write this manuscript.

\section{REFERENCES}

1. Castro, F.E.V.G and Pabico, J.P. (2015) Micro simulations of Arbhing, Clogging, and Bursty exit phenomena in Crowd Dynamics. Proceedings of the 10th National conference on Information Technology Education (NCITE 2012), Laoag, 18-20 October 2012.

2. Lauren Bradshaw, "Crowd Management Plan for an Existing Outdoor Event research Paper", Custom Writing. Com September 30.211 .

3. Poongodi, K., \& Shanthi, J. (2019). Information Seeking Behavior through Crowd sourcing among Students in Selected Arts \& Science Colleges at Salem District affiliated to Periyar University. Qualitative and Quantitative Methods in Libraries, 6(1), 51-69.

4. Sasikumar K., Dhirendra Bhargava, Disaster Management Plan for GIR IGNFA, Dehradun.

5. Zhou, B., Tang, X. and Wang, X. (2015) Learning Collective Crowd Behaviors with Dynamic pedestrian-agents. International Journal of computer vision, 111, 50-68.

6. Spiro G. Doukas, "Crowd Management: Past and Contemporary Issues" The Sport Journal, ISSN: 1543-9518.

7. Dr. R. K. Dave, "India Crowd Management Conference 2017" - the initiative of RESPECT and Gujarat Disaster Management Authority, Ghandinagar.

8. Helbing, D. and Mukerji, P. (2012) Crowd Disasters as Systemic Failures: Analysis of the love parade disaster. EPJ Data Science, 1, 1-40 